bustion engines, fundamental work has been carried out on the mechanism of corrosion inhibition and the rate of oxidation of heat-resistant alloys at high temperatures. Identification of the constituents of Canadian marine and vegetable oils was being continued in part with the view of alternative sources of drying-oils, and laboratory work on a new silver catalyst for the production of ethylene oxide was nearing completion.

In the Division of Physics a Collins liquid-helium cryostat has been installed, while the acoustics group has done much to supply the operational maintenance of the improved marine fog-horn already developed. Using the photographic emulsion technique, a study of very high-energy nuclear disintegrations caused by incoming primary cosmic-ray particles has provided evidence that both neutral and charged mesons are produced in the interaction between such incoming particles and the elementary nucleons (neutrons and protons) which make up atomic nuclei. Using the counter technique an accurate comparison has been made of the intensities of cosmic rays continuously recorded at Ottawa and at Resolute. In the radiology laboratory trial sources of radioactive cobalt have been investigated to obtain information required for the design of radiation therapy equipment using very large radioactive sources, and a method has been developed for measuring the energy output and obtaining the isodose curves of beta-ray plaques used in some types of cancer therapy. Experimental work on the determination of the acceleration due to gravity by direct observation on a falling body continued in the metrology laboratory. The optics laboratory developed a new method, using the Herschel effect, for increasing the effective range of photographic papers, while the spectroscopy group analysed the infra-red spectra of nitrous oxide and heavy methylacetylene and elucidated their molecular structure. In theoretical physics, a systematic study was made of the quantization of the classical theory of charged particles with spin, and the validity of the usual perturbation theory in the auto-ionization of light atoms was studied; calculations of the probabilities of excitation of vibrational states of hydrogen by electrons, protons and hydrogen atoms were made and theories developed for the ferro-electric effect of certain crystals and the dissociation of oxygen molecules in the upper atmosphere.

Housing has continued to dominate the selection of projects for investigation by the Division of Building Research; but jointly with the Division of Chemistry an extensive programme of corrosion research is in operation as well as one on paint failures, and a detailed survey of fire research needs in Canada and of fire research and testing facilities in North America was approaching completion. In the Division of Mechanical Engineering, wind-tunnel studies have been made on four Canadian aircraft, while the work of the model-testing basin was expanded and included tests of naval vessels. Factors affecting ice formation on large bodies of water were studied in connexion with the possibility of maintaining ice-free navigation channels in winter, os well as ice crystal formation in fuels and the operational behaviour of low-temperature lubricants.

The programme of the Radio and Electrical Engineering Division comprised about thirty projects in electrical engineering, electronics, radar and radiophysics. A new marine radar with the minimum range of only ten yards was developed, which gave excellent performance, especially in small harbours, in passing ships at close quarters and in docking; and other developments included a microwave shore beacon for use with a simple inexpensive microwave receiver on board ship, a radio-frequency mass spectrometer which employs no magnetic field, an experimental electron accelerator as a source of betaor gamma-rays with an energy of four million volts, and a new surge generator and a $400-\mathrm{kW}$. pulsegenerator providing extremely bright flashes of light, lasting a few microseconds, at 5-500 times a second. A theoretical study of servo-mechanism performance based on a generalized frequency-response analysis was completed and the infra-red detector used to locate faulty joints on power-transmission lines redesigned. In the Atomic Energy Project, using the electron-pair, neutron and beta-ray spectrometers, new energy-levels have been found in the nuclei of several atoms, and the structure of certain compounds, such as deuterized ammonium chloride and carbon tetrachloride, has been determined. With the new high noutron flux available in the field, new isotopes have been obtained by successive capture of neutrons.

\section{SUMMER DAY-TIME METEOR STREAMS OF 1949 AND 1950}

TNDER the general heading of "The Summer Daytime Meteor Streams of 1949 and 1950", three papers, each dealing with a different aspect of these daylight meteor streams, have recently been published*. The first paper, by A. Aspinall and G. S. Hawkins, describes the method of measurement of the radiant positions and activity. The original single-directional aerial, movable in azimuth, was unsuitable for a general survey of meteor radiants, and a new apparatus has been designed consisting essentially of two fixed, narrow-beamed aerials directed along azimuths $242^{\circ}$ and $292^{\circ} \mathrm{E}$. of $\mathrm{N}$., operating on a wave-length of $4 \mathrm{~m}$. As the radiant of an active shower moves across the sky, it produces echoes in each aerial in turn, and these echoes are recorded by continuous photography; from their range-time variation the right ascension and declination of a major shower can be determined to within $+1 \cdot 5^{\circ}$. Table 2 of this paper gives a comparison of the radiants of four streams from 1947 to 1950 ; these include the $\eta$-Aquarids-the well-known stream established by visual methods and which is supposed to be associated with the debris of Halley's Cometand also the $\beta$-Taurids, the $\zeta$-Perseids and the Arietids. The last two occur simultaneously and wero not successfully interpreted with the single aerial; but the streams have been definitely separated with the new apparatus. In addition to these permanent streams, there are several others which are very active but are of a transient or long-period character, and there are numerous meteors which the apparatus in use fails to assign to any definite radiants.

The second paper, "Measurement of the Velocities", by J. G. Davies and J. S. Greenhow, explains how the radio-echo diffraction technique is used to determine meteor velocities. A description of this apparatus appeared in Nature, 161, 596 (1948), and 8 short account of the method used is given in the papor. Tables 1 and 2 contain the lists of velocities obtained in 1950 and 1949 respectively, and other relevant information. The velocity distribution for

* Mon. Not. Roy. Astro. Soc., 111, No. 1 (1951), communicated by * Mon. Not. Roy. Astro. Soc., 111, No. 1 (1951), communicated by
Prof. A. C. B. Lovell, Jodrell Bank Experimental Station, University Prof. A. C. B. 
each shower shows an appreciable spread about the mean, the significance of which is discussed in the third paper, "Computation of the Orbits", by Mary Almond.

The radiant co-ordinates and volocities of four streams - the o-Cetids in addition to the three others already mentioned-provided the data for the computation of orbits, radiant positions being corrected for zenith attraction and diurnal aberration. Table 2 of the third paper gives the orbital elements and also the duration of the showers, which varied from 8 to 18 days. As might be expected, the radiant position was found to shift from day to day, but as there was no detectable change in the meteor velocities during these periods, the mean radiant positions and the most probable velocity values were used during the time of the activity of each shower. The table gives the spread of the orbital elements, which was estimated by considering the effects of a movement of the radiant within the given limits, and also of the probable spread of the velocity; a short discussion of the spread in each of the orbital elements follows. The orbits of the different showers, projected on the plane of the ecliptic, are shown, all of them being short-period orbits; in the $\beta$-Taurids and Taurids only does the semi-axis major of the orbits exceed 2 astronomical units, and Whipple has associated the latter with Encke's Comet. None of the others has been connected with cometary orbits, but their orbits have a close resemblance to some of those of the minor planets. It is interesting to note that in 1937 Hoffmeister suggested in "Die Meteore" that some meteor streams were associated with planets as well as with comets.

\section{WORK OF THE WORLD HEALTH ORGANIZATION}

$\mathrm{T}$

HE beneficent activities of men of science are many and various; but few are more beneficent than those of the many experts all over the world who serve the United Nations World Health Organization. The Bulletin, the Chronicle and the Technical Reports of the Organization are so well known by now that it is not necessary to direct attention to them. They have, indeed, become so familiar that some of us perhaps fail to appreciate the width of the field they cover and the intensity of labour and the devotion to human welfare that they represent. "The enjoyment of the highest attainable standard of health is one of the fundamental rights of every human being without distinction of race, religion, political belief, economic or social condition." These words, quoted in an address by the deputy directorgeneral of the World Health Organization, Dr. P. Darolle, are taken from the preamble to the constitution of the Organization; and no one can read the successive publications of the Organization without acknowledging gratefully that everyone who serves it, from the director-general to the humblest field-worker, is translating these words into unremit. ting practice all over the world, and that this devoted work is taking effect on a great variety of sources of ill-health. Antibiotics, health statistics, childhood diseases, yellow fever, mental health, hygiene of seafarers, nursing, nutrition, rabies, brucellosis, rickettsioses, tuberculosis and tropical diseases of all kinds-these are only some of the subjects of the growing list of technical reports issued by the Organization. They all represent the work of committees of experts drawn from all over the world. It is perhaps invidious to select any one of these reports for special comment ; but brief notes on four examples of them will indicate their range and value.

Part 1 of a publication entitled "Annual Epidemiological and Vital Statistics, 1939 46"* deals with the vital statistics and causes of death and covers the difficult years 1939-46, when war conditions made the compilation of vital statistics especially difficult; it links up with, and on the whole follows the pattern of, the annual epidemiological reports issued by the Health Organization of the League of Nations, the last volume of which appeared in 1941. This first part supplies, in respect to a number of countries and for the period 1921-46, information on total area, latest available census results, estimated population, birthand fertility-rates, gross and net reproduction-rates and general and infant mortality-rates. In addition, it gives neo-natal mortality-rates for the period 1931-46 and mortality by cause of death in thirtythree countries from the pre-war period until 1946. There is also similar information for large towns. Changes in the revised international lists of causes of death adopted in 1938 are explained.

An example of the work of the World Health Organization taken from another field is the report of "Joint W.H.O./F.A.O. Expert Group on Zoonoses : Report on the First Session" $\dagger$. At this first session, held in Geneva in December 1950, this group defined zoonoses as diseases which are naturally transmitted between vertebrate animals and man, and it discussed bovine tuberculosis, $Q$ fever, anthrax, psittacosis and hydatidosis and their control. The report contains notes on international action for the study and control of other major zoonoses, together with a useful list of these diseases.

More specialized than the above-mentioned works are Nos. $38 \ddagger$ and $39 \S$ of the Technical Report Series. Report No. 38 records the proceedings of the conference of international experts held in December 1950 at Kampala, Uganda, which discussed especially the problems of malaria in Africa, where the chief vectors of malaria are Anopheles gambice and $A$. funestus, the former being much the more important. The other report gives an account of the proceedings of the expert committee which went into session at Kampala immediately after the meetings of this conference. It discusses the malaria policy of the World Health Organization, the control of malaria in Africa south of the Sahara, its control by residual spraying, and also insecticides and strains of insects resistant to them, and the prevention of transport of vectors by aircraft. The therapeutics of malaria is also discussed.

With these and the other reports and publications of the World Health Organization at hand, anyone interested in the world-wide fight against ill-health of all kinds can keep in touch with every aspect of this never-ending war. $\mathrm{H}_{\theta}$ can also see, by reading between the lines, glimpses here and there of the volume of hard work and devotion to human welfare that make all this detailed work possible. There can scarcely be, in any walk of life, a worthier record of human devotion to the welfare of mankind. G. LaPage

* Annual Epidemiological and Vital Statistics. Part 1: Vital Statistics and Causes of Death. Pp. 124. 1951. 20s. $\dagger$ Joint W. F.O.JF.A.O. Expert Group on Zoonoses: Report on the
First Session. (W.H.O. Technical Report Series, No. 40.) Pp. 48 . $1951.2 s .3 d$. $¥$ Malaria Conference in Equatorial Africa Report Series, No. 38.) Pp. 72. 1951. 38. 6d.

\$ Expert Committee on Malaria. Beport on the Fourth Session. (W.H.O. Technical Report Series, No. 39.) Pp. 32. 1951. 1s.6d. (Geneva : World Health Organization; London: H.M.S.O.) 